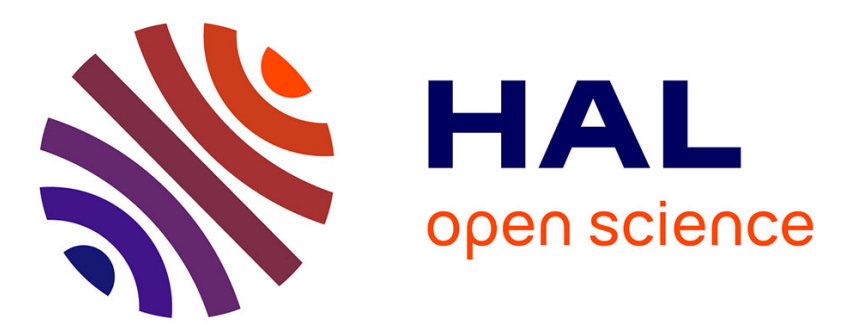

\title{
Finite size scaling study of dynamical phase transitions in two dimensional models: Ferromagnet, symmetric and non symmetric spin glasses
}

\author{
A.U. Neumann, B. Derrida
}

\section{- To cite this version:}

A.U. Neumann, B. Derrida. Finite size scaling study of dynamical phase transitions in two dimensional models: Ferromagnet, symmetric and non symmetric spin glasses. Journal de Physique, 1988, 49 (10), pp.1647-1656. 10.1051/jphys:0198800490100164700 . jpa-00210845

\section{HAL Id: jpa-00210845 https://hal.science/jpa-00210845}

Submitted on 1 Jan 1988

HAL is a multi-disciplinary open access archive for the deposit and dissemination of scientific research documents, whether they are published or not. The documents may come from teaching and research institutions in France or abroad, or from public or private research centers.
L'archive ouverte pluridisciplinaire HAL, est destinée au dépôt et à la diffusion de documents scientifiques de niveau recherche, publiés ou non, émanant des établissements d'enseignement et de recherche français ou étrangers, des laboratoires publics ou privés. 
Classification

Physics Abstracts

05.40

\title{
Finite size scaling study of dynamical phase transitions in two dimensional models : Ferromagnet, symmetric and non symmetric spin glasses
}

\author{
A. U. Neumann $\left({ }^{1,2}\right)$ and B. Derrida $\left({ }^{1}\right)$ \\ ( $\left.{ }^{1}\right)$ Service de Physique Théorique $\left(^{*}\right)$ de Saclay, F-91191 Gif-sur-Yvette Cedex, France \\ $\left({ }^{2}\right)$ Department of Physics, Bar Ilan University, 52100 Ramat Gan, Israel
}

(Reçu le 2 mai, accepté le 3 juin 1988)

\begin{abstract}
Résumé. - Nous étudions l'évolution de deux configurations soumises au même bruit thermique pour plusieurs modèles en dimension 2 : ferromagnétisme et verres de spin symétrique et non symétrique. Pour chacun de ces modèles nous obtenons une température critique non nulle au-dessus de laquelle les deux configurations finissent toujours par se rencontrer. En utilisant les lois d'échelle des systèmes finis, nous déterminons pour ces trois modèles cette transition de phase dynamique et certains exposants critiques. Pour le modèle ferromagnétique, la transition à $T_{\mathrm{c}} \simeq 2,25$ coïncide avec la température de Curie tandis que pour les deux modèles de verre de spin $( \pm J)$ nous obtenons $T_{\mathrm{c}}=1,5-1,7$.
\end{abstract}

\begin{abstract}
We study the time evolution of two configurations submitted to the same thermal noise for several two dimensional models (Ising ferromagnet, symmetric spin glass, non symmetric spin glass). For all these models, we find a non zero critical temperature above which the two configurations always meet. Using finite size scaling ideas, we determine for these three models this dynamical phase transition and some of the critical exponents. For the ferromagnet, the transition $T_{\mathrm{c}} \simeq 2.25$ coincides with the Curie temperature whereas for the two spin glass models ( $\pm J$ distribution of bonds) we obtain $T_{\mathrm{c}} \simeq 1.5-1.7$.
\end{abstract}

\section{Introduction.}

Monte Carlo Methods [1] combined with finite size scaling ideas $[2,3]$ are widely used to study the phase diagrams and the critical properties of all kinds of models in Statistical Mechanics : one first determines as accurately as possible the properties of finite systems (squares, cubes, etc.) of linear size $L$ and then one extracts the critical behaviour of the infinite system by looking at the dependence of the physical properties on the linear size $L$. In the case of a second order phase transition at a critical point $T_{\mathrm{c}}$, one assumes a scaling form valid for large $L$ and in the neighbourhood of $T_{\mathrm{c}}$ : for example the magnetic susceptibility $\chi(L, T)$ of a ferromagnet, which depends on the size $L$ and on the temperature $T$ (and thus is a function of two variables), can be expressed near a critical point $T_{\mathrm{c}}$ and for large $L$ as

$$
\chi(L, T) \sim L^{\gamma / \nu} f\left(L^{1 / \nu}\left(T-T_{\mathrm{c}}\right)\right)
$$

and so becomes a function of a single variable.

(*) Laboratoire de l'Institut de Recherche Fondamentale du Commissariat à l'Energie Atomique.
Recently a new dynamical method based on the comparison of two configurations subjected to the same thermal noise has been proposed. Several dynamical phase transitions were observed using this method for various models (3 dimensional ferromagnet and spin glasses [4], the 2d ANNNI model [5], 2d and $3 d$ automata models [6]). It appears that at high enough temperature a system rapidly forgets its initial condition and consequently two configurations subjected to the same thermal noise become identical very quickly. At low temperature, each configuration may be trapped in a different valley which depends on the initial condition and therefore they remain different for very long. Numerical studies [4-6] as well as analytical calculations done for infinite dimensional models $[7,8]$ show that these two regimes are separated by sharp dynamical phase transitions. An important question is to know how these dynamical phase transitions are related to the usual phase transitions at thermal equilibrium. It is then useful to develop methods capable of giving accurate estimates of the location of these dynamical transitions and of the exponents which characterize the critical behaviours near these transitions. 
In the present paper we use a finite size scaling method to determine these dynamical transitions for several two dimensional models : the $2 \mathrm{~d}$ ferromagnetic Ising model, the $2 \mathrm{~d} \pm J$ spin glass and the $2 \mathrm{~d}$ non symmetric $\pm J$ spin glass (for which $J_{i j}$ and $J_{j i}$ are independent random variables). The main quantity we consider is the time $\tau$ for two configurations to become identical. This time depends on the size and on the temperature and therefore assuming a finite size scaling form similar to (1), we can estimate the transition temperatures and the exponents. The main result of the present work is that the finite size scaling analysis of our numerical data gives a non zero transition temperature for all these $2 \mathrm{~d}$ models. In the case of the ferromagnet, this transition coincides with the Curie temperature of the $2 \mathrm{~d}$ ferromagnet at equilibrium. We also obtain estimates of the critical exponents. These estimates are more accurate in the ferromagnetic case than in the two

$$
\begin{aligned}
& J_{i j}=1 \\
& J_{i j}=J_{j i}=1 \quad \text { with probability } \frac{1}{2} \\
& \left.J_{i j}=J_{j i}=-1 \quad \text { with probability } \frac{1}{2}\right\} \text { for the spin glass } \\
& \left.\begin{array}{ll}
J_{i j}=J_{j i}=1 & \text { with probability } \frac{1}{4} \\
J_{i j}=-J_{j i}=1 & - \\
J_{i j}=J_{j i}=-1 & - \\
J_{i j}=-J_{j i}=-1 & -
\end{array}\right\} \text { for the non symmetric } \\
& \text { for the ferromagnetic case } \\
& \text { for the ferromagnetic case }
\end{aligned}
$$

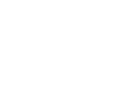

All the calculations in the present work are done using the following parallel dynamics. If the system is in a configuration $\mathrm{C}_{t}=\left\{S_{i}(t)\right\}$ at time $t$, its configuration $\mathrm{C}_{t+1}=\left\{S_{i}(t+1)\right\}$ at time $t+1$ is given by

$$
\begin{aligned}
& S_{i}(t+1)=+1 \quad \text { with probability } p_{i}(t) \\
& \begin{array}{lll}
-1 & - & 1-p_{i}(t)
\end{array}
\end{aligned}
$$

where the $p_{i}(t)$ are given by

$$
p_{i}(t)=\frac{1}{2}+\frac{1}{2} \tanh \left(\sum_{j} \frac{J_{i j} S_{j}(t)}{T}\right) .
$$

By parallel dynamics we mean that at each time step $t$, all the $p_{i}(t)$ are calculated before all the spins $S_{i}$ are updated. The main advantage of parallel dynamics is that the updating of the spins can be easily vectorized in numerical calculations.

We compared, in some tests, parallel dynamics with sequential dynamics (for which during a time interval $\Delta t=1 / N, p_{i}(t)$ for a single spin $i$ is determined and the spin $i$ is updated). We found that spin glass problems because $T_{\mathrm{c}}$ is known exactly for

This paper is organized as follows. In section 2 we describe the stochastic dynamics we use and how we submit two different configurations to the same thermal noise. In section 3, we discuss our finite size sing approach to the problem : this approach is a andizan to dynamics of a method which has . ferromagnetic Ising model. In sections 5 and 6 we repeat the same calculations for the $\pm J$ spin glass and the $\pm J$ non symmetric spin glass.

\section{Dynamics.}

Our calculations are done for Ising spins $\left(\sigma_{i}= \pm 1\right)$ a square lattice of linear size $L$ with periodic $\sigma_{i}$ interact through some nearest neighbour interactions $J_{i j}$ which are given by

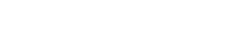

the «physical» times (time for magnetisation to decay, time for two configurations to meet) are longer for parallel dynamics than for sequential dynamics. However, the structure of our programs was such that the sequential dynamics required more computer time than parallel dynamics. Thus we decided to limit our calculations to parallel dynamics.

When one uses parallel dynamics for systems with symmetric interactions $\left(J_{i j}=J_{j i}\right)$, the probability $P\left(\left\{S_{i}\right\}, t\right)$ of finding the system in a configuration $\left\{S_{i}\right\}$ at time $t$ converges to an equilibrium $P_{\text {eq }}\left(\left\{S_{i}\right\}\right)$

$$
\lim _{t \rightarrow \infty} P\left(\left\{S_{i}\right\}, t\right)=P_{\mathrm{eq}}\left(\left\{S_{i}\right\}\right)
$$

which is given by [13]

$$
P_{\mathrm{cq}}\left(\left\{S_{i}\right\}\right)=C \prod_{i=1}^{N} \cosh \left(\frac{\sum_{j} J_{i j} S_{j}}{T}\right)
$$

This probability distribution is different from the usual equilibrium 


$$
\tilde{P}_{\mathrm{eq}}\left(\left\{S_{i}\right\}\right)=C \prod_{1 \leqslant i<j \leqslant N} \exp \left(\frac{J_{i j} S_{i} S_{j}}{T}\right)
$$

which would be obtained by updating the spins in a sequential way (one spin is updated at each time step).

The two equilibrium $P_{\text {eq }}\left(\left\{S_{i}\right\}\right)$ and $\tilde{P}_{\text {eq }}\left(\left\{S_{i}\right\}\right)$ have clearly different properties. If we consider the spins $\left\{S_{i}\right\}$ on a lattice which can be decomposed into two sublattices $\mathrm{A}$ and $\mathrm{B}$ such that any spin of each sublattice interacts only with spins of the other sublattice, we see that (6) gives no correlation between the spins of the two sublattices whereas (7) gives such correlations.

One can prove, however, that these two equilibrium probability distributions ((6) and (7)) have many properties in common. If one decimates (6) and (7) by summing over all the spins of one sublattice (say B), one finds that both (6) and (7) give the same distribution

$$
P^{\mathrm{dec}}\left(\left\{S_{j}\right\}\right)_{j \in \mathrm{A}}=C \prod_{i \in \mathrm{B}} \cosh \left(\frac{\sum_{j} J_{i j} S_{j}}{T}\right) .
$$

This implies that any correlation function $\left\langle S_{j_{1}} \ldots S_{j_{k}}\right\rangle$ of spins located on the same sublattice is the same for (6) and (7). Therefore quantities such as the critical temperature $T_{\mathrm{c}}$, the magnetisation on each sublattice, the correlation length have to be identical for (6) and (7) whereas quantities such as the susceptibility which involve correlations between spins of the two sublattices are different for (6) and (7).

To implement the dynamics (3) and (4), one computes at each time step all the $p_{i}(t)$ using the expression (4) and one updates the spins according to the following formula

$$
S_{i}(t+1)=\operatorname{sign}\left[p_{i}(t)-z_{i}(t)\right]
$$

where the $z_{i}(t)$ are $N$ random numbers uniformly distributed between 0 and 1 .

The method used in the present paper consists in comparing the time evolution of two configurations $\left\{S_{i}(t)\right\}$ and $\left\{S_{i}^{\prime}(t)\right\}$ submitted to the same thermal noise. This means that the two configurations are updated using the same random numbers $z_{i}(t)$ for the two configurations (and the same interactions $\left.J_{i j}\right)$ :

$$
\begin{aligned}
S_{i}(t+1) & = \\
& =\operatorname{sign}\left[\frac{1}{2}+\frac{1}{2} \tanh \left(\frac{\sum_{j} J_{i j} S_{j}(t)}{T}\right)-z_{i}(t)\right]
\end{aligned}
$$

$$
\begin{aligned}
& S_{i}^{\prime}(t+1)= \\
& =\operatorname{sign}\left[\frac{1}{2}+\frac{1}{2} \tanh \left(\frac{\sum_{j} J_{i j} S_{j}^{\prime}(t)}{T}\right)-z_{i}(t)\right] .
\end{aligned}
$$

Clearly if the two configurations become identical at some time $t$, they remain identical for ever.

As in previous works [4, 6-8], the two basic quantities we consider to compare the time evolution of two configurations are :

(1) the survival probability $P(t)$ that two configurations are still different at time $t$;

(2) the distance between the two configurations $\Delta(t)$ defined by

$$
\begin{aligned}
\Delta(t)=\frac{1}{4 N} \sum_{i=1}^{N}\left[S_{i}(t)\right. & \left.-S_{i}^{\prime}(t)\right]^{2}= \\
& =\frac{1}{2 N} \sum_{i=1}^{N}\left|S_{i}(t)-S_{i}^{\prime}(t)\right|
\end{aligned}
$$

$\Delta(t)$ is simply the fraction of spins which are different between the two configurations.

In all the calculations presented here (except for the calculation of the magnetisation of the ferromagnet), we choose the initial configuration $\left\{S_{i}(0)\right\}$ at random and $\left\{S_{i}^{\prime}(0)\right\}$ opposite to it

$$
S_{i}^{\prime}(0)=-S_{i}(0) \text { for all } i \text {. }
$$

We then calculate for each sample $s$, the distance $\Delta_{s}(t)$ defined by (11) and we stop the calculation when the two configurations become identical. We then calculate two moments $\tau_{1}(L, T, s)$ and $\tau_{2}(L, T, s)$ of a characteristic time for the two configurations to meet

$$
\begin{aligned}
& \tau_{1}(L, T, s)=\sum_{t} t \Delta_{s}(t) / \sum_{t} \Delta_{s}(t) \\
& \tau_{2}(L, T, s)=\sum_{t} t^{2} \Delta_{s}(t) / \sum_{t} \Delta_{s}(t)
\end{aligned}
$$

$\tau_{1}$ and $\tau_{2}$ depend of course on the system size $L$, the temperature $T$ and the sample $s$. One can easily see that $\tau_{1}$ and $\tau_{2}$ are measures of characteristic times for the two configurations to meet by taking the example of a pure exponential for $\Delta_{s}(t)$ (if $\Delta_{s}(t)=$ $\exp (-\alpha t)$, then $\tau_{1} \simeq \alpha^{-1}$ and $\left.\tau_{2} \simeq 2 \alpha^{-2}\right)$.

In the next section we will discuss the finite size scaling forms of $\tau_{1}$ and $\tau_{2}$ and we will see that it is convenient to introduce the ratio $R$ defined by

$$
R(L, T, s)=\frac{\tau_{2}(L, T, s)}{\tau_{1}^{2}(L, T, s)} .
$$

Before doing so let us mention that in the case of ferromagnetic interactions (2a), one can measure the magnetisation $m(t)$ from the knowledge of the 
distance $\Delta(t)$ between two configurations. By starting at $t=0$ with two fully polarized configurations

$$
S_{i}(0)=1=-S_{i}^{\prime}(0) \text { for all } i \text {. }
$$

It can be shown [14] that with the dynamics (10) one has at any later time

$$
S_{i}(t) \geqslant S_{i}^{\prime}(t) \text { for all } i
$$

So the distance $\Delta(t)$ defined by (11) can be expressed in the ferromagnetic case and for the initial condition (16) :

$$
\begin{aligned}
\Delta(t)=\frac{1}{2 N} \sum_{i=1}^{N} \mid S_{i}(t) & -S_{i}^{\prime}(t) \mid= \\
= & \frac{1}{2 N} \sum_{i=1}^{N} S_{i}(t)-S_{i}^{\prime}(t) .
\end{aligned}
$$

Averaging over many samples, the distance $\Delta(t)$ gives the average magnetisation $m(t)$ at time $t$ (starting at $t=0$ with $m(0)=1$ ).

\section{Finite size scaling.}

In a high temperature phase, the effect of noise is strong, the two configurations $\left\{S_{i}(t)\right\}$ and $\left\{S_{i}^{\prime}(t)\right\}$ forget quickly their initial conditions and therefore the time $\tau_{1}$ should be short. On the contrary, in a low temperature phase, the two configurations $\left\{S_{i}(t)\right\}$ and $\left\{S_{i}^{\prime}(t)\right\}$ may fall into different valleys and $\tau_{1}$ becomes the activation time to jump over the barriers which separate these valleys; one then expects $\tau_{1}$ to increase as the exponential of a power law $L^{x}$ of the size $L$ ( $L^{x}$ is the typical height of a barrier. At the transition temperature $T_{\mathrm{c}}$ between these two regimes, it is reasonable to think that $\tau_{1}$ has a finite size scaling form

$$
\tau_{1}(L, T, s) \sim L^{z} f_{1}\left(L^{1 / \nu}\left(T-T_{\mathrm{c}}\right), s\right)
$$

which should be valid for $L \gg 1$ and $\left|T-T_{\mathrm{c}}\right| \ll 1$. In (19) we have left the sample dependence of $\tau_{1}$. This means that $\tau_{1}$ as defined up to now is sample dependent and the meaningful quantity to consider is the probability distribution of $\tau_{1}$.

In principle by computing averages of $\tau_{1}$ or of functions of $\tau_{1}$ (i.e. $\log \tau_{1}$ ) over many samples for several sizes and temperatures, one can try to determine the three unknown $z, \nu$, and $T_{\mathrm{c}}$ in (19). In practice it is more convenient to consider a quantity like $R$ defined in (15) which has a simple finite size scaling. Since $\tau_{2}$ is also a measure of a squared characteristic time for two configurations to meet, one expects that

$$
\tau_{2}(L, T, s) \sim L^{2 z} f_{2}\left(L^{1 / \nu}\left(T-T_{\mathrm{c}}\right), s\right)
$$

and therefore

$R(L, T, s)=\frac{\tau_{2}(L, T, s)}{\tau_{1}^{2}(L, T, s)}=f_{3}\left(L^{1 / \nu}\left(T-T_{\mathrm{c}}\right), s\right)$.

If one averages $R$ (or functions of $R$ ) over many samples $s$, one finds that

$$
\langle R(L, T, s)\rangle=g_{3}\left(L^{1 / \nu}\left(T-T_{\mathrm{c}}\right)\right)
$$

or

$$
\langle\log R(L, T, s)\rangle=\tilde{g}_{3}\left(L^{1 / \nu}\left(T-T_{\mathrm{c}}\right)\right) .
$$

We see from (22) and (23) that $T_{\mathrm{c}}$ can be determined from the data for several sizes. One draws $\langle R\rangle$ or $\langle\log R\rangle$ versus $T$ for several sizes $L$ and the point where the curves, corresponding to different sizes, cross gives $T_{\mathrm{c}}$. A similar approach based on the ratio of moments of the magnetization has been often used to study the critical point of ferromagnets $[9$, 12].

Since the finite size scaling forms (22) and (23) are expected to be valid only for large $L$, one expects the curves corresponding to different $L$ to cross at the same points only for large enough sizes.

Once $T_{\mathrm{c}}$ has been estimated, one can try to determine the exponent $\nu$ by looking at how $\frac{\mathrm{d}\langle R(L, T, s)\rangle}{\mathrm{d} T}$ depends on the size $L$ at $T_{\mathrm{c}}$ and $z$ by measuring the slope of $\log \left\langle\tau_{1}\left(L, T_{\mathrm{c}}, s\right)\right\rangle$ (or of $\left.\left\langle\log \tau_{1}\left(L, T_{\mathrm{c}}, s\right)\right\rangle\right)$ versus $L$.

At $T_{\mathrm{c}}$, one can also study the full time dependence of the distance $\Delta_{s}(t)$ defined by (11). $\Delta_{s}(t)$ depends on the time $t$, the size $L$, the temperature $T$ and the sample $s$. If one averages $\Delta_{s}(t)$ over many samples at $T=T_{\mathrm{c}}$, one gets a quantity $\delta(L, t)$ :

$$
\delta(L, t)=\left\langle\Delta_{s}(L, t)\right\rangle
$$

which depends on the size $L$ and on time. For $t \gg 1$ and $L \gg 1$, one expects a finite size scaling form

$$
\delta(L, t) \sim L^{-a} f\left(t / L^{z}\right)
$$

We will show in the next sections our results for $\delta(L, t)$ and we will extract from our data estimates of the exponents $a$ and $z$.

In the case of the ferromagnet, a similar finite size scaling form is expected for the average magnetisation $m(t)$ at $T_{\mathrm{c}}$ :

$$
m(L, t) \sim L^{-b} f\left(t / L^{z}\right)
$$

and again the exponents $b$ and $z$ can be estimated by assuming this scaling form. In the case of the $2 \mathrm{~d}$ ferromagnet, the critical exponents at thermal equilibrium are known exactly $(\nu=1, \beta=1 / 8$ ) (here $\beta$ is the exponent of the magnetisation below $T_{\mathrm{c}}$ ) and one expects that $b=\beta / \nu=0.125=1 / 8$. 


\section{The 2d ferromagnet.}

In this section we present the results of our numerical calculations in the case of the 2 dimensional ferromagnetic Ising model. We did our calculations for $L=10,20$ and 40 and the number of samples generated was respectively 10000,2000 and 500. So the number of spins was roughly the same for all the sizes. For each sample, the initial condition was a random configuration $\left\{S_{i}(0)\right\}$ and the other configuration $\left\{S_{i}^{\prime}(0)\right\}$ opposite to it. All samples were iterated until the distance $\Delta_{s}(t)$ between the two configurations has vanished.

In figure 1 we show $\langle R\rangle$ defined by (15) and (22) and $\langle\log (R)\rangle$, as a function of temperature for the three different sizes. One can see that the three curves cross almost at the same point which can be estimated from figure 1 to be $T_{\mathrm{c}}=2.25 \pm 0.05$. This value agrees with the exact Curie temperature $T_{\mathrm{c}}=2.269$ of the $2 \mathrm{~d}$ Ising model. This result shows that studying the distance $\Delta_{s}(t)$ is a possible way of determining $T_{\mathrm{c}}$ for a ferromagnetic system [14]. We did not try to measure the ratio $R$ below $T_{\mathrm{c}}$ because the time for two configurations to become identical
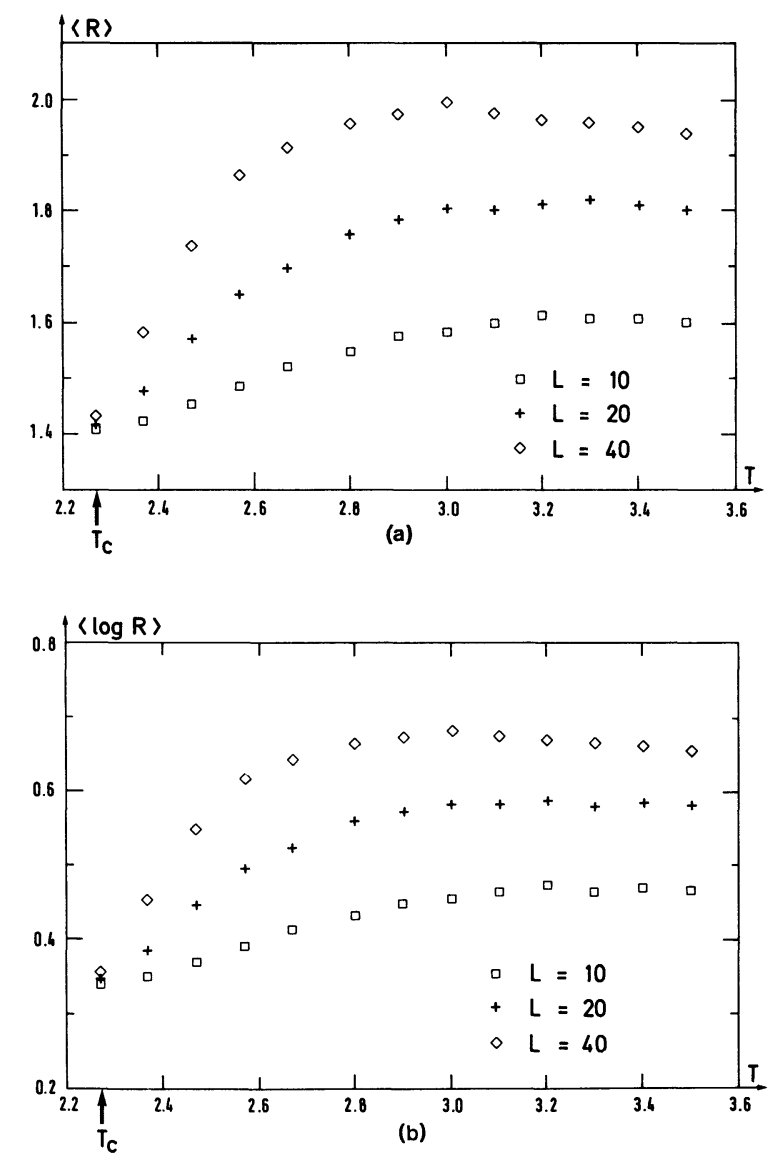

Fig. 1. - (a) The ratio $\langle R\rangle=\left\langle\tau_{2} / \tau_{1}^{2}\right\rangle$ versus $T$ for different sizes $L$ for the $2 \mathrm{~d}$ ferromagnet. The curves corresponding to different $L$ meet at $T_{\mathrm{c}}$. (b) The same as figure 1a for $\langle\log R\rangle$. increases very rapidly as $T$ decreases and so does our computer time.

For $T=T_{\mathrm{c}}=2.269$, we show in figure 2 the survival probability $P(t)$ which is the fraction of samples $s$ such that $\Delta_{s}(t) \neq 0$. We see that as $L$ increases, it takes longer and longer for the two configurations to meet (for $L=40$, there remains $1 / 3$ of the samples after $10^{4}$ time steps and it took almost $10^{5}$ time steps for the distance to vanish in all our 500 samples). As this time grows roughly like $L^{2}$, we had to limit ourselves to $L \leqslant 40$.

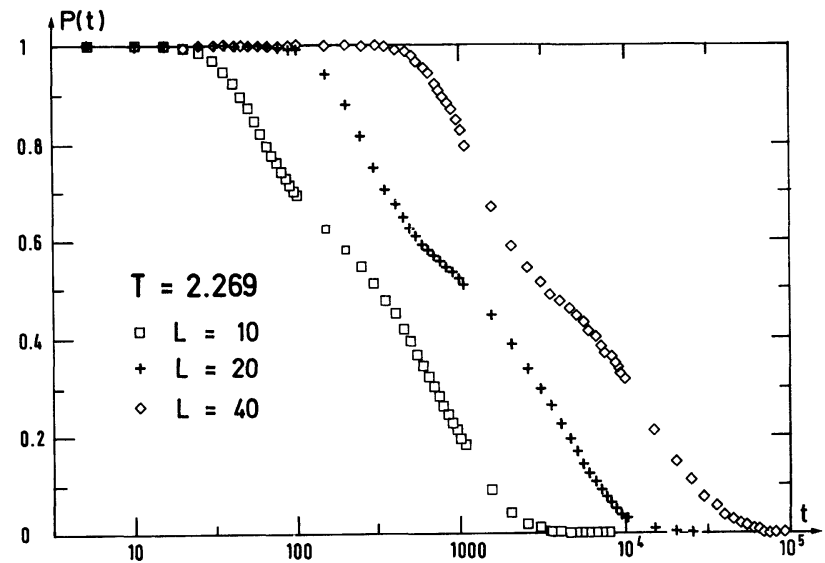

Fig. 2. - The survival probability $P(t)$ versus $t$ for the $2 \mathrm{~d}$ ferromagnet at $T=T_{\mathrm{c}}$.

In figure 3a we show the average distance $\delta(L, t)$ defined in (24) for the $2 \mathrm{~d}$ ferromagnet at $T=T_{\mathrm{c}}$. The behaviour seems to be a power law at short times, a cross over region and a faster decay at long times. As $L$ increases, the cross over occurs later.

In figure $3 \mathrm{~b}$ we show $L^{a} \delta(L, t)$ versus $t / L^{z}$ in a $\log$-log plot. We see that for $a=0.26$ and $z=2.1$, our results are consistent with the finite size scaling form (25). Trying to vary the exponents $a$ and $z$ we found that for

$$
\begin{aligned}
& a=0.26 \pm 0.02 \\
& z=2.10 \pm 0.05
\end{aligned}
$$

the curves corresponding to the three sizes ( $L=10,20,40$ ) looked superimposed.

We did not find a way of relating the exponent $a$ to already known exponents. For the exponent $z$ our estimate (27) agrees rather well with previous numerical determinations of the dynamical exponent $z$ [15].

In figure 4 we show the average magnetisation $m(L, t)$ measured as explained in section 2 by choosing for the initial condition $\left\{S_{i}(0)=1\right\}$ and $\left\{S_{i}^{\prime}(0)=-1\right\}$. Again we see a power law at times shorter than a cross over time when the decay becomes faster. Our results are consistent with the 

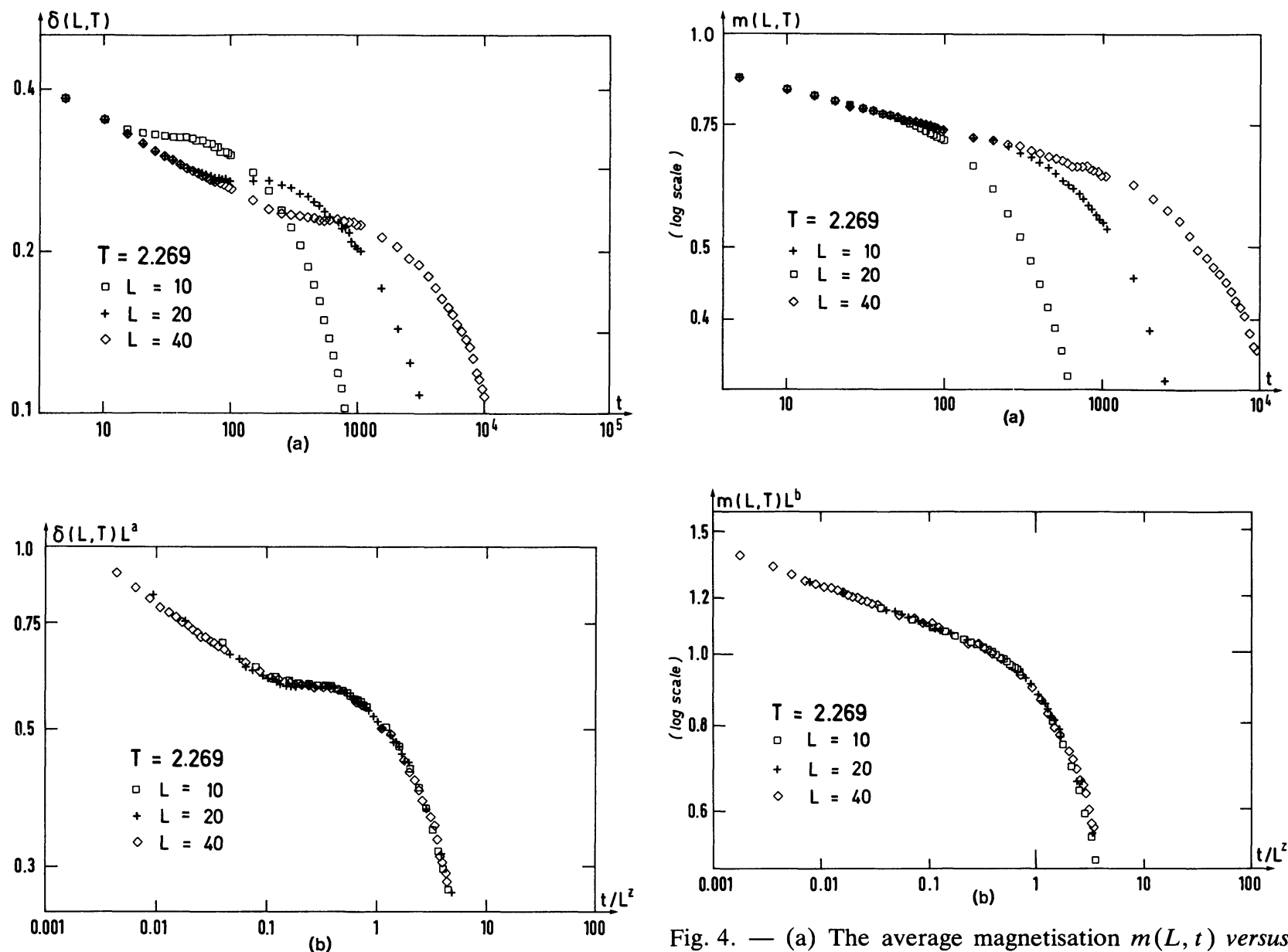

Fig. 4. - (a) The average magnetisation $m(L, t)$ versus $t$ (log-log plot) fordifferentsizes $L$ for the $2 \mathrm{~d}$ ferromagnet. (b)

Fig. 3. - (a) The average distance $\delta(L, t)$ versus $t$ (log$\log$ plot) for different sizes $L$ for the $2 \mathrm{~d}$ ferromagnet at $T_{\mathrm{c}}$. (b) $L^{a} \delta(L, t)$ versus $t / L^{z}(\log -\log$ plot) for $a=0.26$ and $z=2.1$. These data are consistent with the finite scaling form (25).

scaling form (26) with

$$
\begin{aligned}
& b=0.125 \pm 0.005 \\
& z=2.10 \pm 0.05 .
\end{aligned}
$$

So $b$ takes the value $\beta / \nu$ as expected and we recover the same estimate for $z$ as in (27).

We see that for the $2 \mathrm{~d}$ ferromagnetic Ising model, one can estimate the transition temperature by studying the ratio $R$ and that one can measure the exponent $z$ from the time dependence of either the distance $\delta(L, t)$ or the magnetisation $m(L, t)$. From our results we can also predict the power law decay of $\delta(t, L)$ and $m(t, L)$ for times $T \ll L^{z}$ :

$$
\begin{aligned}
& \delta(t, L) \sim t^{-\frac{a}{z}} \\
& m(t, L) \sim t^{-\frac{b}{z}}
\end{aligned}
$$

where $a, b$ and $z$ are given by (26) and (27). $z=2.1$.

\section{The symmetric $\pm J$ spin glass.}

Using the same method, the same sizes and the same number of samples as for the ferromagnet case, we try now to find the critical temperature $T_{\mathrm{c}}$ and the critical exponents $a$ and $z$ for the symmetric $\pm J$ spin glass defined by (2b). Here, since the interactions are random, we changed also the interactions for each new sample.

In figures $5 \mathrm{a}$ and $5 \mathrm{~b}$ we show $\langle R\rangle$ and $\langle\log R\rangle$ versus $T$ for several sizes $L$. We can estimate from these figures

$$
T_{\mathrm{c}}=1.6 \pm 0.1 .
$$

The accuracy is worse than in the ferromagnetic case because the point where $\langle R(L, T)\rangle$ and $\left\langle R\left(L^{\prime}, T\right)\right\rangle$ cross depends more on $L$ and $L^{\prime}$ than in the ferromagnetic case. So in the case of spin glasses, the finite size effects seem stronger, and it would be necessary to increase the size $L$ in order to improve the accuracy of $T_{\mathrm{c}}$.

Since $T_{\mathrm{c}}$ is not known very accurately, we expect rather large error bars in the estimates of the critical 


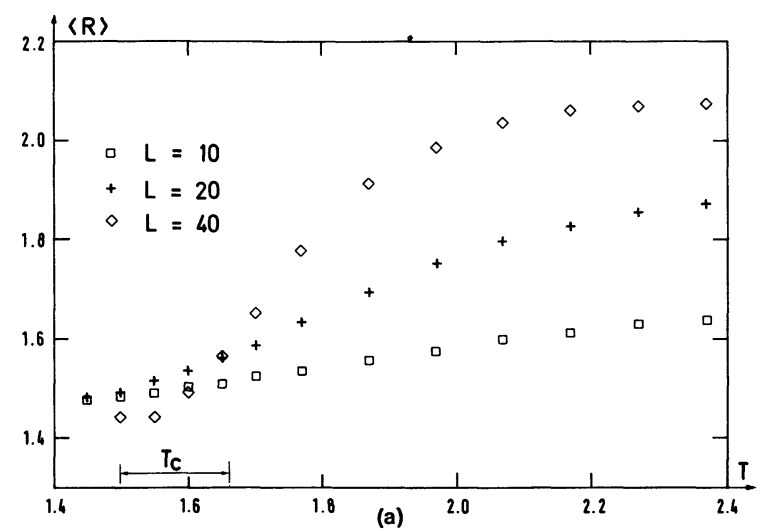

Table I. - Exponents $a$ and $z$ for different values of a chosen critical temperature $T_{\mathrm{c}}$, for the case of symmetric $\pm J$ spin glass. $z_{1}$ and $z_{2}$ are the slopes of $\left\langle\log \tau_{1}\right\rangle$ as function of $\log L$ as defined in equation (31). $z_{3}$ an a are estimated by trying to satisfy the best the scaling form (25).

\begin{tabular}{ccccc}
\hline$T_{\mathrm{c}}$ & $z_{1}$ & $z_{2}$ & $z_{3}$ & $a$ \\
\hline 1.6 & 2.15 & 3.04 & $2.6 \pm 0.5$ & \\
\hline 1.65 & 1.92 & 2.30 & $2.1 \pm 0.3$ & \\
\hline 1.7 & 1.73 & 1.66 & $1.70 \pm 0.05$ & \\
\hline 1.77 & 1.48 & 1.12 & $1.3 \pm 0.2$ & \\
\hline
\end{tabular}

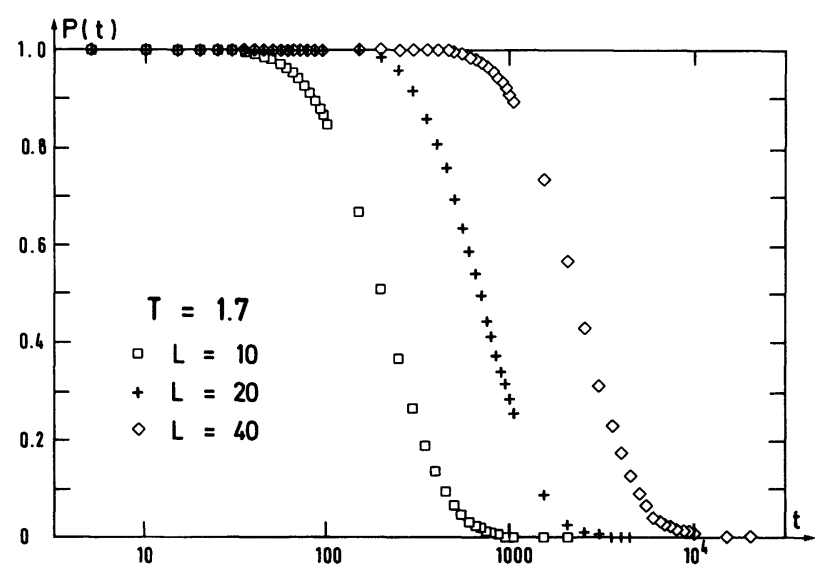

exponents $z$ and $a$. In table I, we give estimations $z_{1}$ and $z_{2}$ of $z$ obtained by the following formula

$z_{1}=\frac{\left\langle\log \tau_{1}(T, 2 L, s)\right\rangle-\left\langle\log \tau_{1}(T, L, s)\right\rangle}{\log 2}$

for $L=10$, and $z_{2}$ is given by the same expression for $L=20$.

We see that the values of $z$ vary with what we choose for $T_{\mathrm{c}}$ but the results seem not to depend much on $L$ for $T_{\mathrm{c}} \simeq 1.7$ leading to an estimate for $z \simeq 1.7$

We calculated the survival probability and the average distance $\delta(L, T)$ for various temperatures in the range $1.5 \leqslant T_{\mathrm{c}} \leqslant 1.7$. We found that the finite size scaling form (25) was better satisfied for $T=1.7$ and we will present our data only for that temperature.

In figure 6 , we show the survival probability as a function of time at $T=1.7$. One can see that the characteristic time for two configurations to meet increases roughly like $L^{1.7}$ with $L$.

Figures (7a) and (7b) give the average distance $\delta(L, T)$ for $T=1.7$. When we tried to plot $\delta(L, T) \quad L^{a}$ versus $t / L^{z}$, we found that for

Fig. 6. - The survival probability versus $t$ for the $2 \mathrm{~d} \pm J$ symmetric spin glass model at $T=1.7$.

$z=1.70 \pm 0.05, a=0.65 \pm 0.07$, the curves corresponding to the three sizes were superimposed.

Of course if we change $T$, we have to change the value of $z$ and of $a$. In table I, we give $z_{3}$ and $a$ obtained by trying to superimpose the curves for different $L$ when $T$ is changed. We see that $z_{3}$ changes a lot with $T$ whereas the value of $a$ is much more stable. Comparing with $z_{1}$ and $z_{2}$ we see again that at $T_{\mathrm{c}}=1.7$, the $z$ estimates of $z \simeq 1.7$ seem to coincide.

Although $T_{\mathrm{c}}$ and the exponents $z$ and $a$ cannot be estimated as accurately as in the ferromagnetic case, the finite size scaling analysis of our results gives a strong evidence of a finite $T_{\mathrm{c}} \simeq 1.7$ for the $\pm J$ spin glass model.

We have already seen in $3 \mathrm{~d}$ [4] that the dynamical transition studied in the present paper occurs at a much higher temperature than the temperature where a spin glass phase appears. So in $2 \mathrm{~d}$, a dynamical transition at $T=1.7$ does not contradict 

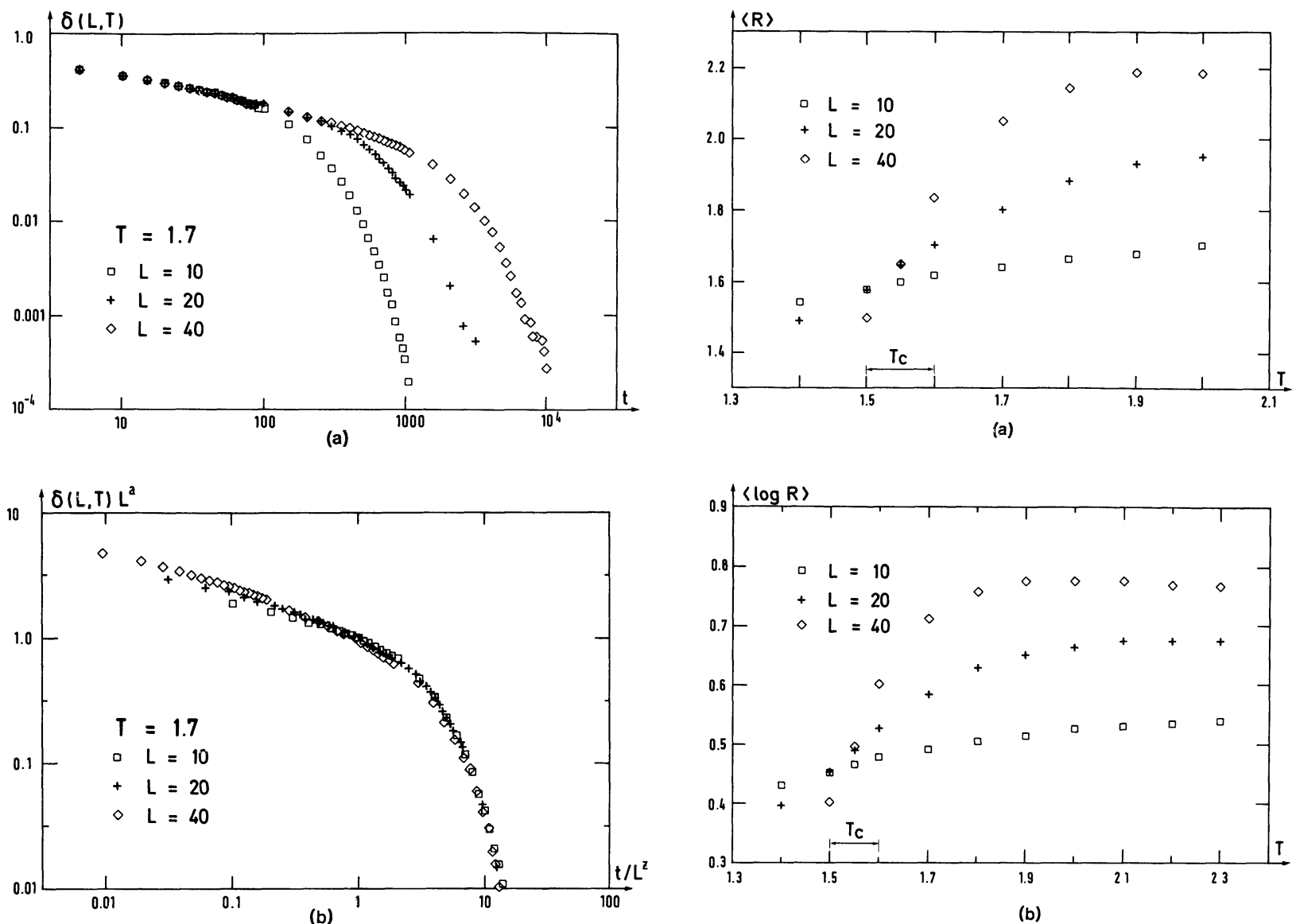

(b)

Fig. 7. - (a) The average distance $\delta(L, t)$ versus $t$ (log$\log$ plot) at $T=1.7$ for the $\pm J$ symmetric spin glass model. (b) $L^{a} \delta(L, t)$ versus $t / L^{z}$ (log-log plot) for $a=0.65$ and $z=1.7$.

the presently accepted idea that there is no spin glass phase at finite temperature [16].

It is however possible that when one crosses $T_{\mathrm{c}} \simeq 1.7$, some relaxation processes become much slower; a simple picture for the divergence of $\tau_{1}$ at $T_{\mathrm{c}}$ could be that below $T_{\mathrm{c}}$, there exist some infinite barriers in phase space which prevent the two configurations from meeting.

\section{The non-symmetric $\pm J$ spin glass.}

Using, again, the same method, sizes and number of samples as for the previous cases we try to find the critical temperature $T_{\mathrm{c}}$ and the critical exponents $a$ and $z$ for the non symmetric $\pm J$ spin glass defined by (2c).

In figures $8 \mathrm{a}$ and $8 \mathrm{~b}$ we show $\langle R\rangle$ and $\langle\log R\rangle$ versus $T$ for several sizes $L$. From these figures we estimate that

$$
T_{\mathrm{c}}=1.56 \pm 0.06
$$

The accuracy of this estimate is better than for the case of symmetric spin glass although it is not as good as for the ferromagnetic case.

Fig. 8. - (a) and (b) : The same as figures 1 and 5 in the case of the $2 \mathrm{~d}$ non symmetric $\pm J$ spin glass. The curves corresponding to $L=10,20$ and 40 cross in the region $T=1.55 \pm 0.05$ with a tendency for $T_{\mathrm{c}}$ to increase with $L$.

It is also interesting to note that the time needed for the distance to disappear is smaller in this case than in the first two cases, as can be seen in figure 9, which shows the survival probability $P(t)$ as function of time for $T_{\mathrm{c}}=1.55$.

Figure 9 and the average distance $\delta(L, T)$ as function of time plotted in figure $10 \mathrm{a}$ are shown at

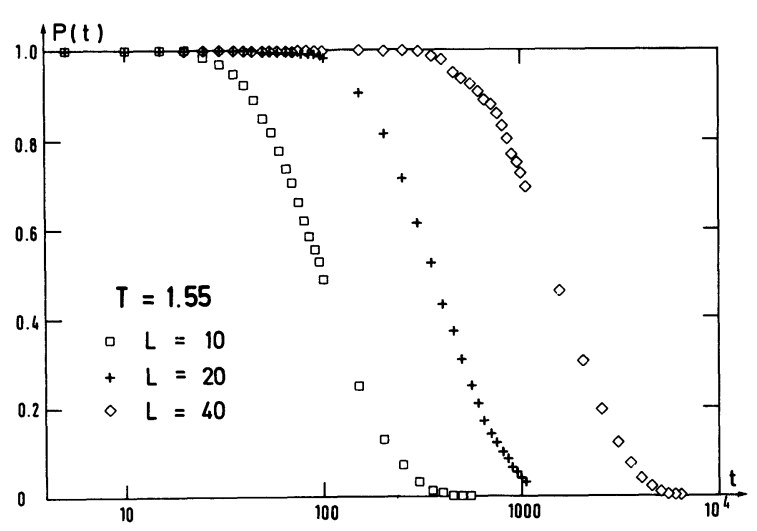

Fig. 9. - The survival probability versus $t$ for the $2 \mathrm{~d} \pm J$ non symmetric spin glass model at $T=1.55$. 

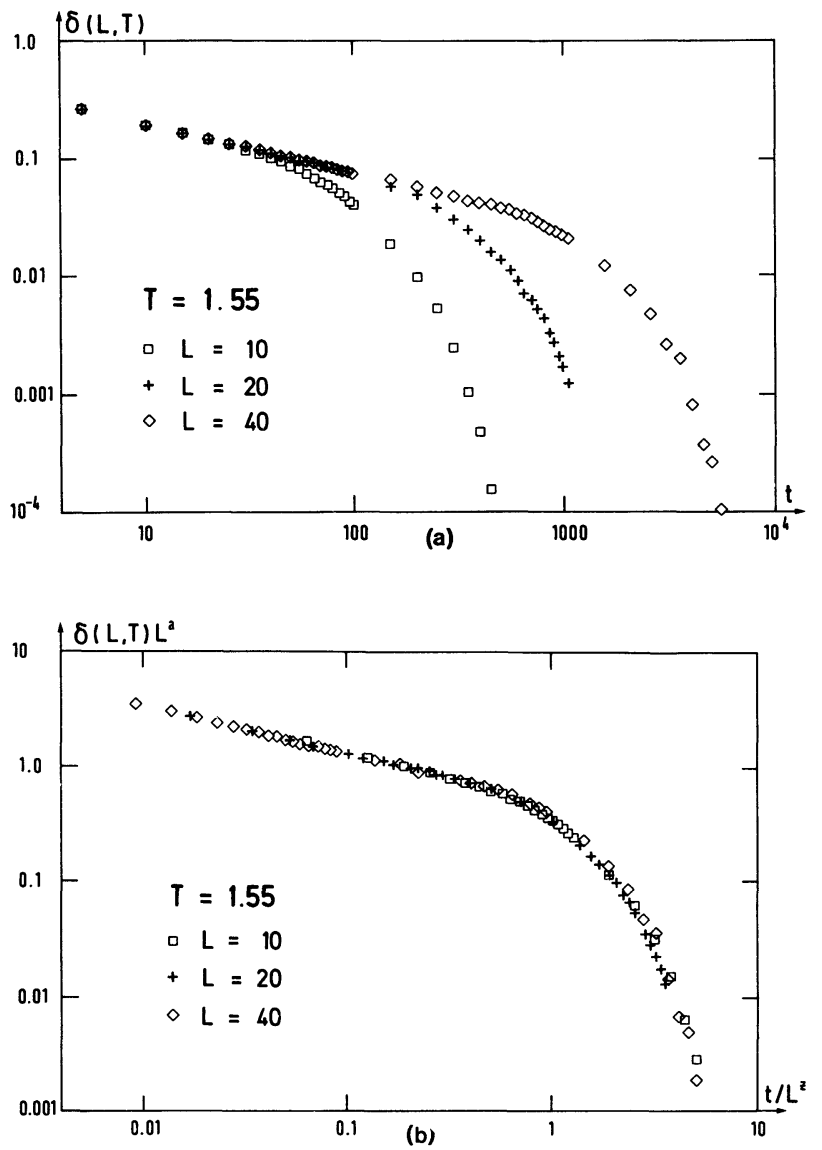

Fig. 10. - (a) The average distance $\delta(L, t)$ versus $t$ (log-log plot) at $T=1.55$ for the $\pm J$ non symmetric spin glass model. (b) $L^{a} \delta(L, t)$ versus $t / L^{z}$ (log-log plot) for $a=0.77$ and $z=1.9$.

Table II. - Exponents a and $z$ for different values of a chosen critical temperature $T_{\mathrm{c}}$, for the case of non symmetric $\pm J$ spin glass. $z_{1}$ and $z_{2}$ are the slopes of $\left\langle\log \tau_{1}\right\rangle$ as function of $\log L$ as defined in equation (31). $z_{3}$ and $a$ are estimated by trying to satisfy the best the scaling form (25).

\begin{tabular}{ccccc}
\hline$T_{\mathrm{c}}$ & $z_{1}$ & $z_{2}$ & $z_{3}$ & $a$ \\
\cline { 1 - 4 } 1.5 & 2.26 & 3.24 & $2.75 \pm 0.5$ & \\
\cline { 1 - 4 } 1.55 & 1.81 & 2.03 & $1.9 \pm 0.1$ & $0.77 \pm 0.05$ \\
\hline 1.6 & 1.51 & 1.14 & $1.3 \pm 0.3$ & \\
& & & &
\end{tabular}

$T_{\mathrm{c}}=1.55$ because it is at this temperature that our data for the three sizes $(L=10,20$ and 40$)$ satisfy the best the scaling form (25), as can be seen in figure $10 \mathrm{~b}$ for the exponents $\alpha=0.77 \pm 0.05$ and $z=1.9 \pm 0.1$.

But, like in the symmetric spin-glass case, $T_{\mathrm{c}}$ is not known very accurately and the estimate of the exponent $z$ depends on the value chosen for $T_{\mathrm{c}}$. So, we give in table II the exponents $a$ and $z_{3}$ estimated by trying to superimpose the curves for the three different sizes at temperatures betwwen 1.5 and 1.6.

We also give in table II the exponents $z_{1}$ and $z_{2}$ defined in equation (31) (the slope of a log-log of $\left\langle\tau_{1}\right\rangle$ as function of $L$ ). It is seen that also $z_{1}$ and $z_{2}$ give the closest result for $T_{\mathrm{c}}=1.55$.

\section{Conclusion.}

For all the two dimensional models studied here (ferromagnet, symmetric $\pm J$ spin glass, non symmetric $\pm J$ spin glasses) we have observed a dynamical phase transition when we compare the time evolution of two configurations submitted to the same thermal noise. For the ferromagnet, this dynamical phase transition coincides with the Curie temperature $T_{\mathrm{c}}^{\mathrm{F}}=2.269$ whereas for the two spin glass models we find $T_{\mathrm{c}} \simeq 1.5-1.7$ which is clearly much lower than $T_{\mathrm{c}}^{\mathrm{F}}$ (excluding the idea that this $T_{\mathrm{c}}$ could be related to the appearance of Griffiths singularities). We have shown how finite size scaling ideas can be used to study these dynamical phase transitions and we have obtained estimates of the critical exponents at these transitions.

We think that the approach we have proposed here could be used in longer calculations to improve our estimates of $T_{\mathrm{c}}$ and of the exponents. Of course, the main question would be to know for the spin glass models what is the change in the landscape of phase space which occurs when one crosses $T_{\mathrm{c}}$ and wether this transition can be recovered by studying quantities $[18,19]$ other than the distance between two configurations.

\section{Acknowledgments.}

This research has benefited of a partial financial support from the BRAIN program of the ECC (grant ST 2J-0422-C).

\section{References}

[1] Binder, K., Monte Carlo Methods in Statistical Physics (1979) ; Applications of the Monte Carlo Method in Statistical Physics (1984) (Springer, Berlin, Heidelberg, New York).

[2] Binder, K., Ferroelectrics 73 (1987) 43.
[3] Barber, M. N., Phase Transitions and Critical Phenomena Eds. Domb and Lebowitz Vol. 8 (1983).

[4] Derrida, B., Weisbuch, G., Europhys. Lett. 4 (1987) 657. 
[5] Barber, M. N., Derrida, B., J. Stat. Phys. 51 (1988) 877.

[6] Derrida, B., Stauffer, D., Europhys. Lett. 2 (1986) 739 ;

de Arcangelis, L., J. Phys. A 20 (1987) L369.

[7] Golinelli, O., Derrida, B., 49 (1988) 1663.

[8] Derrida, B., J. Phys. A 20 (1987) L271.

[9] Bruce, A. D., J. Phys. C 14 (1981) 3667.

[10] Binder, K., Z. Phys. B 43 (1981) 119.

[11] Barber, M. N., Pearson, R. B., Richardson, J. L., Toussaint, D., Phys. Rev. B 32 (1985) 1720.

[12] Saleur, H., Derrida, B., J. Phys. France 46 (1985) 1043.
[13] Fontanari, J. F., Köberle, R., Phys. Rev. A 36 (1987) 2475 ; J. Phys. France 49 (1988) 13.

[14] Lebowitz, J. L. (1987) private communication.

[15] Williams, J. K., J. Phys. A 18 (1985) 49 and references therein.

[16] Binder, K., Young, P., Rev. Mod. Phys. 58 (1986) 801 and references therein.

[17] Griffiths, R. B., Phys. Rev. Lett. 23 (1969) 17.

Randeria, M., Sethna, J. P., Palmer, R. G., Phys. Rev. Lett. 54 (1985) 1321.

[18] Ogielsky, A., Phys. Rev. B 32 (1985) 7384.

[19] Sourlas, N., J. Phys. Lett. France 45 (1984) L-969 ; Europhys. Lett. 1 (1986) 189 ; preprint (1988). 